\title{
3D MODELLING, SIMULATION AND PREDICTION OF FACIAL WRINKLES
}

\author{
Sokyna Al-Qatawneh ${ }^{1}$, Ali Mehdi ${ }^{2}$, and Thamer Al Rawashdeh ${ }^{3}$ \\ ${ }^{1}$ Department of Multimedia Systems, Faculty of Science and IT, Al-Zaytoonah \\ University of Jordan, Amman, Jordan, S . Qatawneh e zuj . edu • jo \\ ${ }^{2}$ Department of Computing and Iinformatics, Faculty of IT, Saudi \\ ElectronicUniversity, Riyadh, Saudi Arabia, A.Mehdi@seu.edu.sa \\ ${ }^{3}$ Department of Computer Science, Faculty of Science and IT, Al-Zaytoonah \\ University of Jordan, Amman, Jordan, Thamer. R@zuj . edu. jo
}

\begin{abstract}
Ageing is a natural phenomenon and it is part of our daily life process; the Facial Ageing (FA) process has been of a great interest to many researchers and some firms like airports and police departments; this is due to the fact that the face appearance changes as people age resulting in difficulties identifying certain individuals. In this paper, two-dimensional wrinkle maps will be used in the design of a three-dimensional system for the purpose of facial wrinkles simulation and prediction. Our findings will challenge many commercial softwares in the innovation of the techniques in setting solid grounds to generate real-time $3 D$ wrinkles that can be used later for various reasons that may include security, 3D facial ageing simulation systems, facial animation, etc. The $2 D$ binary wrinkles will be mapped on the corresponding $3 D$ face models using the generated outlined images. NURBS curves will then be projected on those wrinkles to form a three-dimensional wrinkle map. The coloured wrinkle map, as well as some parameters, will be combined together in an algorithm to predict the appearance of the individual wrinkles in every age group that are divided into decades, starting from the age of 20. The novelty of the adopted procedure in comparison to the previous works, is the new elements that have been integrated and collaborated to boost accuracy and to generate a more realistic outcome.
\end{abstract}

\section{KEYWORDS}

Facial Aging; Facial Wrinkles; Wrinkle Maps; 3D Wrinkle Modelling.

\section{INTRODUCTION}

Facial Ageing (FA) process has been of a great interest to many researchers and some firms like airports and police departments; this is due to the fact that the face appearance changes as people age resulting in difficulties identifying certain individuals. FA research is a form of Facial Recognition (FR) and the main reason for facial recognition is delivered from the forensic needs to identify victims or suspects[1]. The human brain has the ability and capacity to quickly distinguish between known and unknown faces. The biggest advantage of using the face (over 
other parts of the human body) in many applications is that wherever a person may be, the face is also there.

Moreover, the face carries a significant number of features that identify individuals such as gender, emotional state, ethnic origin, age etc. The available facial ageing simulation and prediction technologies lack in simulation accuracy, especially when simulating the appearance of wrinkles. This might be due to the fact that wrinkles appear in different locations on human faces and the amount of change in their development also differs. Failing to simulate the wrinkles in real-time can have a direct impact on the ageing prediction accuracy as wrinkles play a vital role as a major ageing feature.

One of the most popular 2D facial ageing simulation systems is the April Age Progression Software 'age-me'[2]. On the other hand, FaceGen [3] is a widely known 3D face generator modelling software that includes an embedded ageing system. Both softwares are available for commercial purposes and share the disadvantage of generating wrinkles based on assumptions and no real data is considered. Our findings will challenge both commercial softwares in the innovation of the techniques in setting solid grounds to generate real-time $3 \mathrm{D}$ wrinkles that can be used later for various reasons that may include security, 3D facial ageing simulation systems, facial animation, etc.

This work will commence by generating a 3D face model for any given individual. 2D binary wrinkles will be mapped on the corresponding 3D face models using the generated outlined images. NURBS curves will then be projected on those wrinkles to form a three-dimensional wrinkle map. The coloured wrinkle map, as well as some parameters, will be combined together in an algorithm to predict the appearance of the individual wrinkles in every age group that are divided into decades, starting from the age of 20.

In the following section a review of related literature will be presented, while section 3 explainsthe work methodology, in which the 3D models are acquired, as well as the 3D face mesh processing, texture mapping and wrinkles construction are discussed. The results of the simulations and predictions are presented in section 4. Finally, section 5 will conclude the findings on the 3D modelling, simulation and prediction of facial wrinkles.

\section{RELATED WORK}

In addition to the fact that facial wrinkle appearance is a vital sign of ageing, it also plays an important feature in presenting FE; therefore, it became the focus of a number of FE research.Yin and Basu [4] presented a partial texture updating method for facial expression synthesis with facial wrinkles. Based on the observation of the wrinkle appearance and the change along with performed expressions, they extracted the partial texture information, then used the texture dissimilarity between the neutral expression and the active expression to extract the active texture for the expression representation.

For expressive facial animations, Li et al. [7] developed a method for modelling dynamic facial wrinkle. They started with structured facial mesh, then divided the face region into wrinkle subregions, and created some wrinkle lines with their algorithm in these sub-regions. Some key nodes were labelled on the facial mesh; these key nodes affect wrinkle lines if wrinkle lines located the sub-regions that abut on the key nodes. With the algorithm, motion of wrinkle lines 
was produced by movement of the key nodes. Consequently, wrinkle lines derived facial mesh to model expressive wrinkles.

From expressions on a 3D face model, Antini et al. [8] proposed a framework for threedimensional face representation and matching for identification purposes. Basic traits of a face were encoded by extracting curves (wrinkles) of salient ridges and ravines from the surface of a dense mesh. A compact graph representation was then extracted from these curves through a modelling technique that was capable to quantitatively measure spatial relationships between curves in a three-dimensional space. Therefore, face recognition was obtained by matching 3D graph representations of faces.

\section{METHODOLOGY}

\subsection{D Face Creation}

The construction of 3D face models using 2D face photographs, such as the work in [9], has become the focus of study on 3D face modelling, in which generated textures will affect directly the sense of reality of the generated 3D face models[10] and it is indeed one of the most difficult problems in the fields of computer graphics and computer vision.

On the other hand, 3D scanners are becoming widely employed for the task of 3D face generation. However, in this work, the 3D face models are generated from the neutral face of any individual using FaceGen for its simplicity and relative low cost.

The 2D face image is marked (Figure 1) for feature point extraction then processed. The method is based on a 3D morphable face model that encodes shape and texture in terms of model parameters. The stages of this process are shown in Figure 2, where the 3D face model as well as the facemask (texture) are exported.

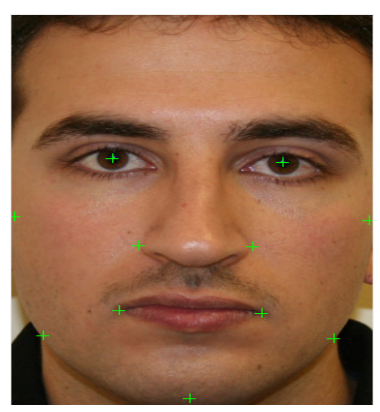

Figure 1: Marked 2D face image

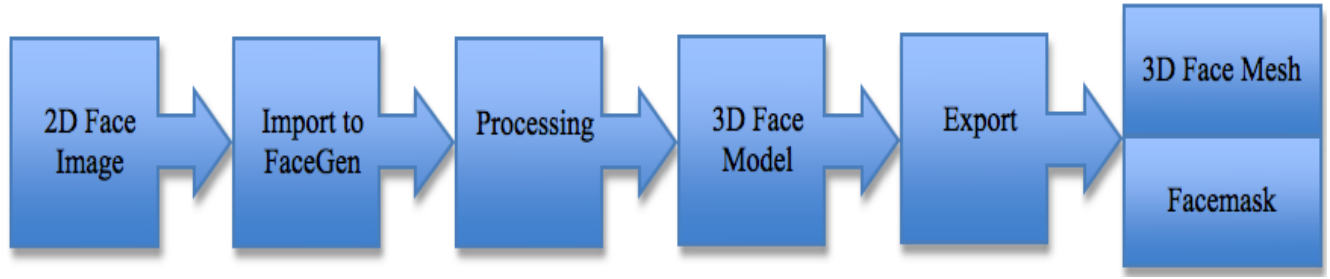

Figure 2: The process of generating and exporting the 3D face model 
The process of the image takes about $10-15$ minutes depending on the speed of the system. The result of the 3D face model is displayed in Figure 3 while the exported materials are shown in Figure 4.

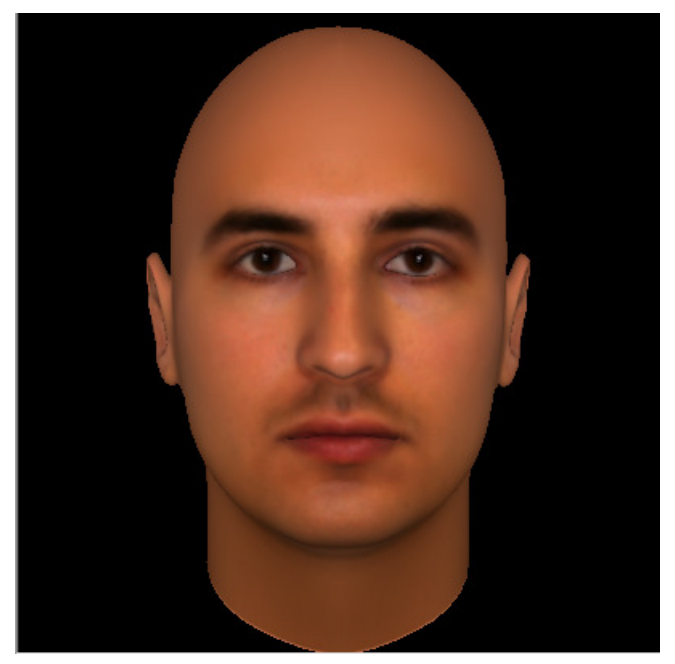

Figure 3: The 3D face model

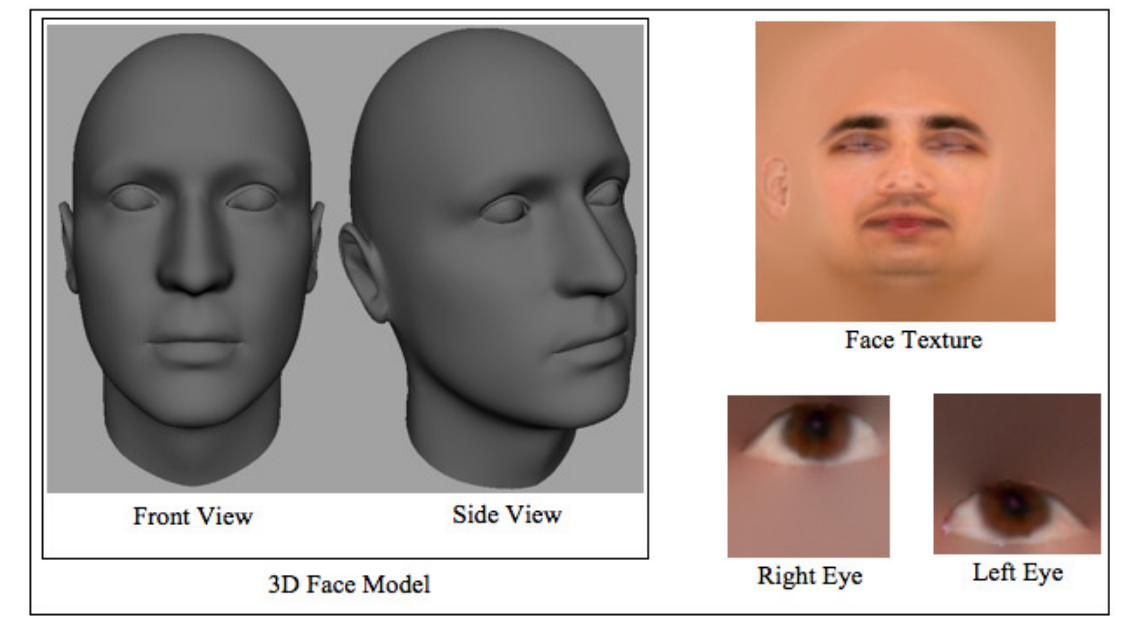

Figure 4: Exported items

\subsection{The 3D Face Mesh}

The generated face models contain simple mesh; in the modelling process of wrinkles, we take into consideration the flow of the facial muscles, so that the edge loops are in the direction of the natural flow of the human muscles (as described in Figure 5). 


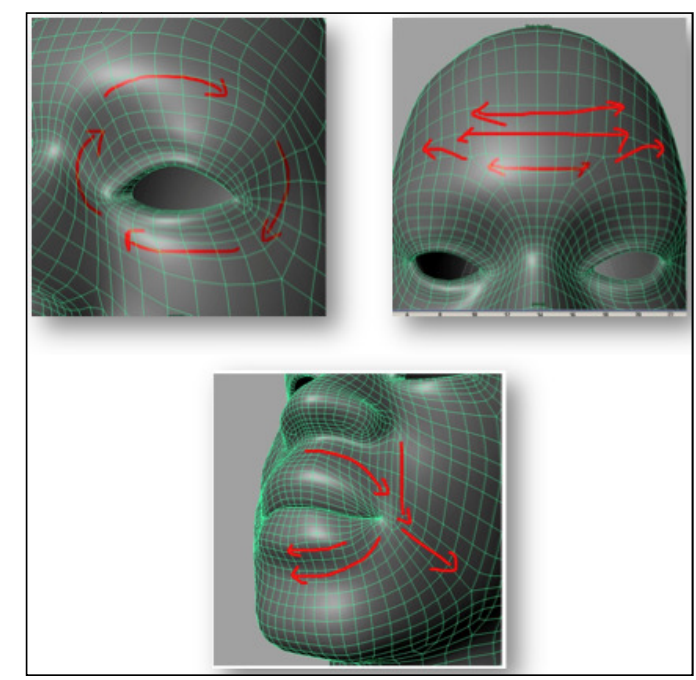

Figure 5: Facial muscle flow

The 3D face has to be processed further for optimisation; this process involves increasing the mesh by adding edge loops in order to boost the smoothness of the wrinkles later in the simulation. This method might cause the program to have a slow loading time due to the addition of more geometry to the model.

An alternative method to create wrinkles can be implemented by generating a so-called normal map $[11,12]$. In 3D computer graphics, normal mapping is a technique used for faking the lighting of bumps and dents. It is used to add details without using more polygons,

We fabricated our own normal map (an example is shown in Figure 6); the depressions make the surface look deeper, which gives the illusion of wrinkles. In our work, accuracy and realistically in terms of the wrinkle locations are top priorities; hence, using normal maps will be inadequate. For this reason, the higher resolution 3D face polygons will be used in this work despite the contingency of a slow loading time.

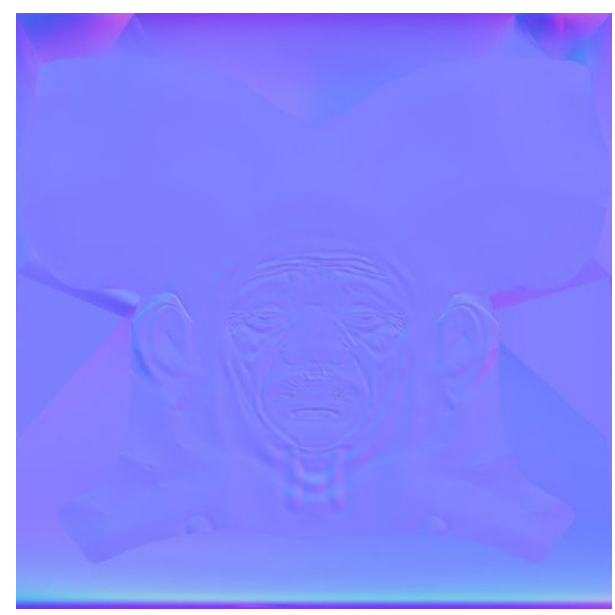

Figure 6: Our generated normal map 


\subsection{Texture Mapping}

Texture mapping is the method for adding detail or colour to a computer-generated graphics or 3D Models. In this section, the texture that is generated in Figure 4 is considered using a UV space. The UV mapping is the process of texturing a 2D image on a 3D object. In contrast to $\mathrm{X}, \mathrm{Y}$ and $\mathrm{Z}$, which are the coordinates for the original 3D object in the modelling space, another set of coordinates is required to describe the surface of the mesh, so the letters $U$ and $V$ are used. The Figure 7 shows an example of our 3D faces that is opened up to be a UV map.

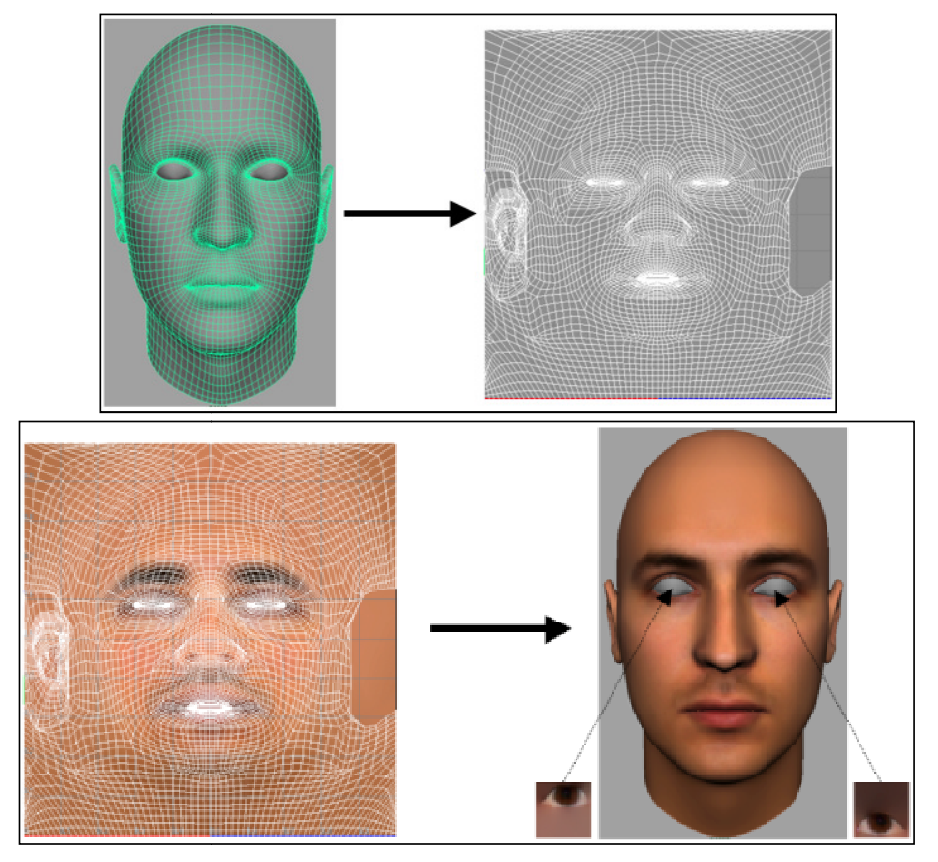

Figure 7: (a) Our 3D face and its UV map; (b) Textured 3D head model

The eyes are textured in the same manner but on a smaller scale.The final texturing result is presented in the figure 8 .

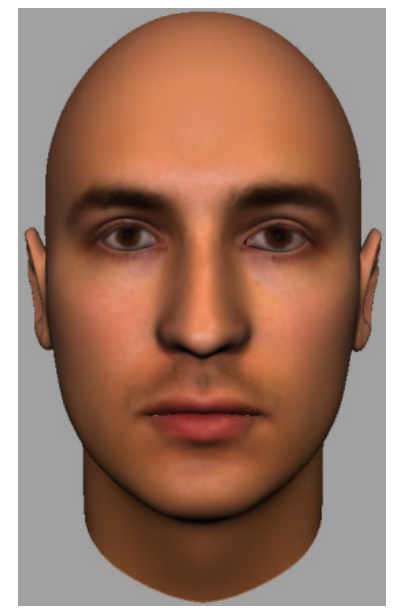

Figure 8: Final texture 


\subsection{Wrinkles Construction}

The generated binary wrinkle maps are mapped on their corresponding 3D face models; Figure 9 illustrates an example. Wrinkles are then constructed on the 3D models by the projection of the NURBS curves.

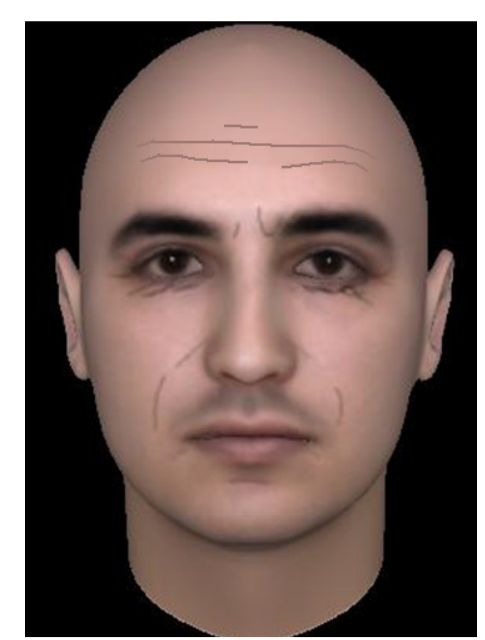

Figure 9: The binary wrinkle map is textured on the 3D face model

Upon the projection of the NURBS curves; the curves are modelled to enhance the look of the 3D wrinkle maps (Figure 10). This is performed by observing the original images in order to add or fill gaps that might be present in the binary wrinkle maps due to detection failure.

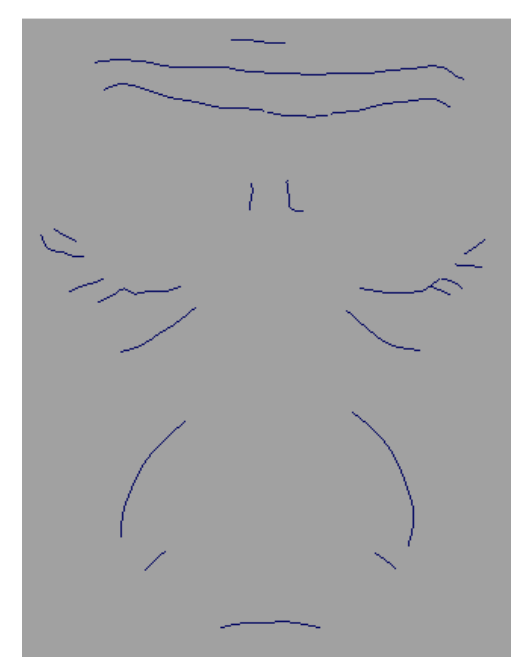

Figure 10: An enhanced 3D wrinkle map 


\subsection{Wrinkles Simulation and Prediction}

For the tasks of simulation and prediction, three elements (for any individual) are considered: the binary wrinkle map, the general development of the wrinkles over the decades and the coloured wrinkle map.

The binary map acts as a benchmark that displays the full facial wrinkles and their locations. While the coloured map will work within each age group, i.e. the wrinkles that have the highest intensity values will appear first and so on. Finally, the findings will be displayed in a form of a graph to study the behaviour of the wrinkles as age progresses.

\section{RESULTS}

Some sample results are presented in figure 11 and compared to the simulation results that are generated by the commercial software FaceGen. Our results are presented on the left hand side while FaceGen results are presented on the right hand side.

Our method doesn't only work on the standard head models that are taken from FaceGen, but it accepts 3D face scans as well. Figures 12 are generated results using a 3D face scan for Person S and also compared to the generated results from FaceGen:

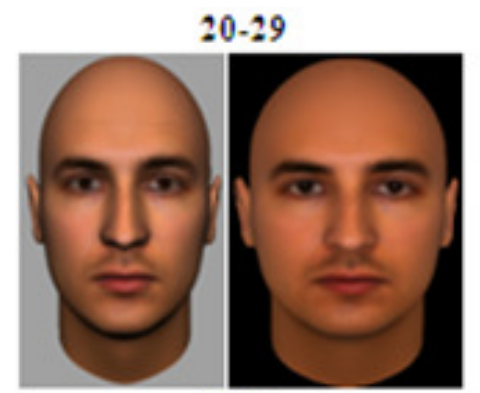

$30-39$

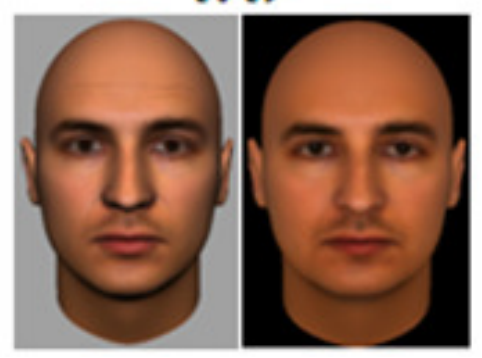

$40-49$

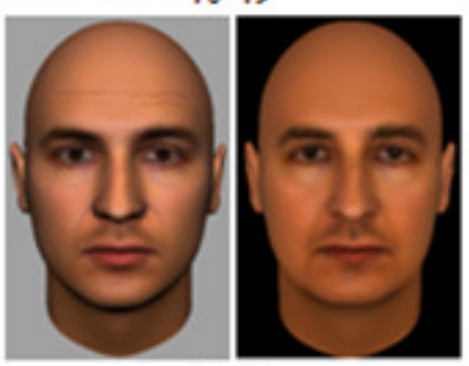

$50-59$

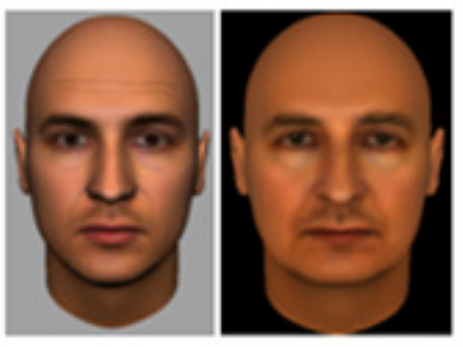

Figure 12: A comparisons between our proposed system results(on the left hand side) and FaceGen results (on the right hand side).

\section{DISCUSSION AND CONCLUSION}

In this work, novel techniques were used in the tasks of facial wrinkles simulation and prediction. Various parameters were utilised in order to develop this unique facial wrinkle pattern formation 
system, which include the generated wrinkle maps for any given individual along with published literature on the general development of the facial wrinkles over the years.

The provided system has the privilege of using real-time data that has high potential to boost the accuracy. Moreover, the system was compared to other available popular technologies and proved competence.
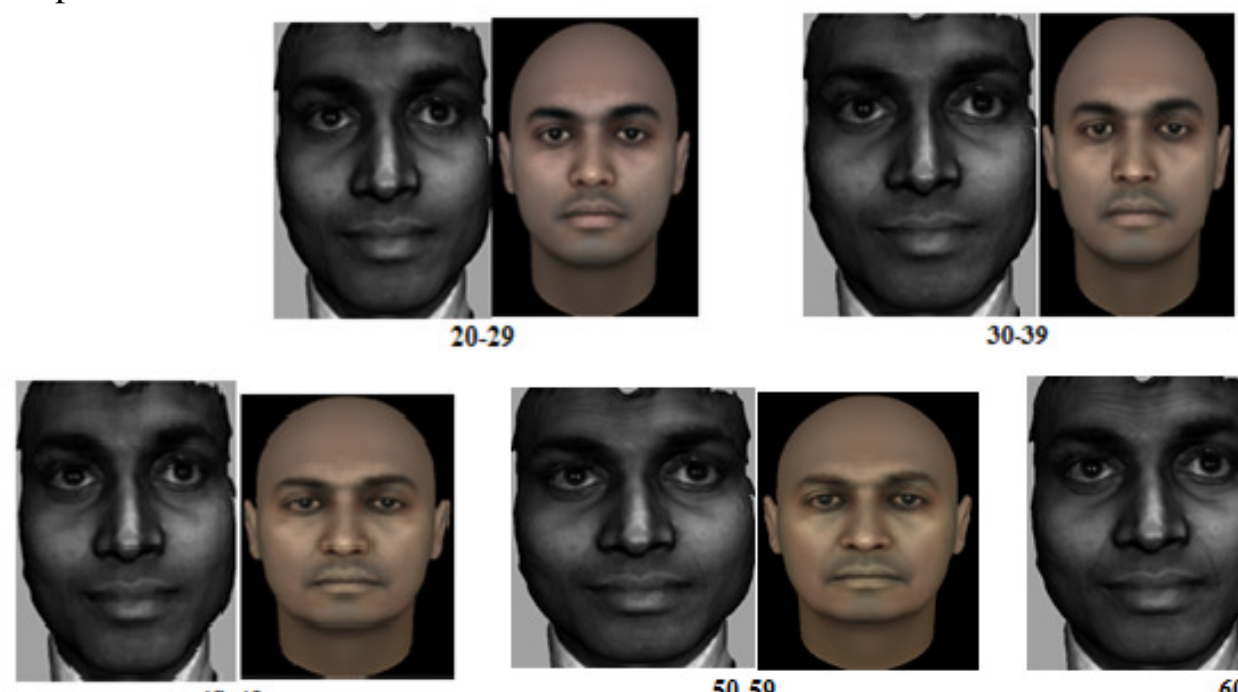

40-49

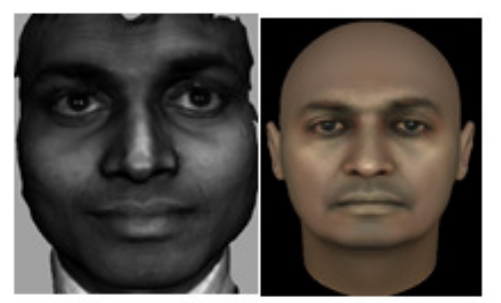

$50-59$

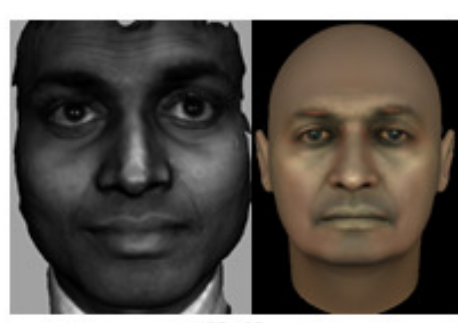

$60-69$

Figure 12: A comparisons between generated results using a 3D face scan results (on the left hand side) and FaceGen results (on the right hand side).

In comparison to FaceGen, it was seen from their results that their system could only display common facial wrinkles; Better results than FaceGen were obtained by using the age-me software. This software uses 2D images and performs the age predictions using 2D approach. The generated wrinkles in this software do not match the actual wrinkle maps of the individuals, which shows that either some assumptions were made in the generation of wrinkles based on data estimation or wrinkles were randomly produced. Though, both cases jeopardise the accuracy of wrinkles formation.Furthermore, the developed system has the distinctive advantage of its ability to accept standard (mean) 3D head models as well as 3D face scans.

\section{REFERENCES}

[1] Clement, J., and Marks, M., "Computer Graphic Facial Reconstruction". 2005, USA: Elsevier.

[2] April Age Progression Software. [cited 17/01/2011]; Available from: http://www.age-me.com/.

[3] FaceGen. [cited 31/03/2010]; Available from: http://www.facegen.com/.

[4] Yin, L., and Basu, A., "Generating Realistic Facial Expressions with Wrinkles for Model-Based Coding". Computer Vision and Image Understanding, November 2001. Vol. 84(Issue 2): p. 201-240.

[5] Zhang, Y., "Muscle-Driven Modeling of Wrinkles for 3D Facial Expression", in IEEE international conference on multimedia and expo. 2008, IEEE: Hannover. p. 957-960.

[6] Tian, Y., Kanade, T. and Cohn, J. , "Recognizing action units for facial expression analysis", in IEEE Transactions on Pattern Analysis and Machine Intelligence. 2001, IEEE. p. 97-115.

[7] Li, M., Yin, B., Kong, D. and Luo, X. , "Modeling Expressive Wrinkles of Face For Animation", in Proc. of Fourth International Conference on Image and Graphics 2007, IEEE. p. 874-879. 
[8] Antini, G., Berretti, S., Del Bimbo, A., and Pala, P., "3D Face Identification Based on Arrangement of Salient Wrinkles", in IEEE International Conference on Multimedia and Expo. July 2006: Toronto, Ont. p. 85-88.

[9] Lin, W., Weijun, H., Rui, C., and Xiaoxi, W., "Three-Dimensional Reconstruction of Face Model Based on Single Photo", in Proceedings of the International Conference on Computer Application and System Modeling 2010, IEEE: Taiyuan. p. 674.

[10] Wang, H., Zheng, Q., and Sun, Y., "A Method of Generating Global View Texture Images in 3D Face Modeling", in The sencond International Conference on Information Science and Engineering (ICISE). December 2010, IEEE: Hangzhou, China. p. 3435.

[11] Cohen, J., Olano, M., Manocha, D., "Appearance-Preserving Simplification", in Proceedings of the 25th annual conference on Computer graphics and interactive techniques. 1998, ACM: New York, USA.

[12] Yang, B., Pan, Z., "A Hybrid Adaptive Normal Map Texture Compression Algorithm", in Proceedings of the 16th International Conference on Artificial Reality and Telexistence--Workshops. 2006, IEEE: Hangzhou. 